\title{
An Intervention for Treating Alcohol Dependence: Relating Elements of Medical Management to Patient Outcomes With Implications for Primary Care
}

\author{
Denise B. Ernst, $P b D^{1}$ \\ Helen M. Pettinati, $P b D^{2}$ \\ Roger D. Weiss, $M D^{3}$ \\ Dennis M. Donovan, $\mathrm{PbD}^{4}$ \\ Richard Longabaugh, EdD \\ ${ }^{1}$ Center on Alcoholism, Substance Abuse, \\ and Addiction, University of New Mexico, \\ Albuquerque \\ ${ }^{2}$ Treatment Research Center, University \\ of Pennsylvania, Philadelphia \\ ${ }^{3}$ Harvard Medical School, McLean \\ Hospital, Belmont, Massachusetts \\ ${ }^{4}$ Alcoholism and Drug Abuse Institute, \\ University of Washington, Seattle \\ ${ }^{5}$ Center for Alcohol and Addictions Study, \\ Brown University, Providence, Rhode Island
}

Conflicts of Interest: Dr Ernst bas no potential, perceived or real conflict of interest. Dr Pettinati bas received grant/research support from Alkermes, AstraZeneca, Bristol-Myers Squibb, Cepbalon, Forest Laboratories, and Ortho-McNeil. She has served as consultant and on the advisory board and speaker's bureau for Alkermes, AstraZeneca, Bristol-Myers Squibb, Cepbalon, Forest Laboratories, and Ortho-McNeil. Dr Weiss serves on the board of advisers to Alkermes and has received research support from Ortho-McNeil. Drs Donovan and Longabaugh have no potential, perceived, or real conflict of interest.

\section{CORRESPONDING AUTHOR}

Denise Ernst, PhD

Center on Alcoholism, Substance Abuse, and Addiction

2650 Yale Ave SE, MSC11-6280

Albuquerque, NM 87106

dernst@unm.edu

\begin{abstract}
PURPOSE Alcohol dependence, frequently seen in medical settings, is a major problem that affects the health and well-being of many individuals and their families. The purpose of this study was to examine the relationship between treatment outcomes and patient and clinician factors specifically associated with a medically oriented intervention given for the treatment of alcohol dependence. The intervention was developed for the National Institute on Alcohol Abuse and Alcoholism-sponsored COMBINE Study, a randomized controlled trial combining 2 medications, naltrexone and acamprosate, with Medical Management, with or without specialty alcohol treatment.
\end{abstract}

METHODS We examined the effect of patient adherence to treatment (number of Medical Management visits, total minutes in treatment, alliance or therapeutic relationship with the clinician, patient satisfaction with treatment, and clinician adherence to the Medical Management protocol) on abstinence from alcohol, amount of heavy drinking, and clinical improvement during treatment.

RESULTS More Medical Management visits attended and less total time spent in Medical Management treatment was associated with more days of abstinence from alcohol, reductions in heavy alcohol drinking, and a higher likelihood of clinical improvement. The patients' positive perceptions of their alliance with their clinician and their satisfaction with treatment was significantly associated with more days of abstinence from alcohol during treatment. Two clinician factors clinician confidence in the Medical Management treatment and flexibility in delivering Medical Management were also associated with better patient outcomes.

CONCLUSIONS Medically trained clinicians with minimal specialty training in alcohol dependence treatments were able to deliver a brief and effective medication management intervention that was designed to be consistent with primary care practice.

Ann Fam Med 2008;6:435-440. DOI: 10.1370/afm.884

\section{INTRODUCTION}

$\Lambda$ though persons with alcohol disorders frequently receive treatment in specialty programs, they more commonly are cared for in general health care settings, where they receive treatment for other medical problems. A growing literature suggests that incorporating treatment for alcohol problems into primary care can potentially promote better health outcomes and result in major benefits, including financial savings and more comprehensive care. ${ }^{1-6}$ In addition, using effective medications and managing alcohol treatment in primary care may increase the availability of treatment for patients who would normally go without such care.

The purpose of this study was to examine the contributions of clinician and patient variables associated with a medical management approach that 
was found useful in treating alcohol dependence with naltrexone. The data examined are from the National Institute on Alcohol Abuse and Alcoholism (NIAAA)sponsored COMBINE study of 1,383 treatmentseeking outpatients with alcohol dependence. This randomized controlled trial compared 9 combinations of pharmacotherapies and behavioral interventions for alcohol dependence. Results from $\mathrm{COMBINE}^{7}$ suggested that patients given a particular type of medical management (Medical Management) with naltrexone significantly reduced their drinking compared with the other treatment groups. In addition, patients receiving the Medical Management intervention with placebo pills had better drinking outcomes than patients receiving no pills and only the behavioral treatment from an alcohol treatment specialist. Details of the study rationale and design are available elsewhere. ${ }^{8,9}$

Medical Management approximates a primary care approach to alcohol dependence and supports the use of pharmacotherapy. Elements of specialty behavioral treatments, such as motivational interviewing and skills training, were excluded because they require training not typically found in primary care settings. ${ }^{10,11}$ During the initial visit, the clinician reviews the patient's assessments, highlighting symptoms of alcohol dependence and the need for treatment. The patient is advised to stop drinking, educated about alcohol dependence, provided a rationale for medication, and instructed on the importance of daily medication adherence. The clinician and patient also jointly develop an individualized medicationadherence plan; the patient is encouraged to attend support groups and is given information on the medications. At follow-up visits, drinking behavior and medication adherence are ascertained, and plans for reducing drinking or achieving abstinence are revised as needed.

We hypothesized that patients' attendance in treatment, perceptions of their relationship with the health care clinician during treatment, and satisfaction with treatment would be associated with the level of drinking during treatment and predictive of clinical outcomes at the end of treatment. ${ }^{12,13}$ Additional patient variables, including the total face-to-face time with clinicians and number of clinicians seen during treatment, were evaluated. The contribution of the clinician's professional background, degree of involvement with Medical Management patients, and clinician performance during delivery of Medical Management were examined in relation to patient drinking and clinical outcomes.

\section{METHODS}

\section{Participants and Setting}

This analysis was based on the 1,226 (89\% of the $1,383)$ patients randomized to receive Medical Man- agement. Two patients had incomplete demographic data, resulting in a sample of 1,224 (377 women and 847 men). Median age was 44 years, $71 \%$ had fewer than 12 years of education, $42 \%$ were married, and $24 \%$ were ethnic minorities.

Patient inclusion in the COMBINE study required a diagnosis of alcohol dependence, a pattern of regular heavy drinking, and 4 days of abstinence before randomization. Exclusion criteria included a recent history of drug dependence (other than nicotine), psychiatric disorders requiring medication, and unstable medical conditions (including liver enzymes levels of greater than 3 times normal). ${ }^{9}$ Women who were pregnant, nursing, or at risk of pregnancy through inadequate contraception were excluded.

In the 30 days before treatment, patients averaged 20 days of heavy drinking, 13 drinks per drinking day, and 7.5 days of abstinence from alcohol.

The COMBINE study took place at 11 clinical sites, all of which were affiliated with academic alcohol research programs.

\section{Medical Management Schedule and Quality Monitoring}

The intervention consisted of 9 patient visits scheduled during the 4 months of treatment with medications, one at randomization, and the remaining at weeks 1, 2, 4, 6, $8,10,12$, and 16 . The Medical Management manual ${ }^{10}$ is available through the NIAAA Web site (http://pubs. niaaa.nih.gov/publications/combine/). A description of the development, implementation, professional and educational requirements, and training and supervision procedures of clinicians participating in the Medical Management intervention is given in Pettinati et al. ${ }^{11}$

Briefly, the clinicians were medically trained professionals with at least 2 years of postdegree experience. They were expected to have a basic working knowledge of the medications used and medication adherence factors, as well as some familiarity with alcohol dependence. Clinicians completed standardized training that included an overview of the treatment, procedures, and documentation. They submitted samples of structured practice based on case scenarios to demonstrate their competence in the style and skills of the intervention required for certification. Ongoing clinical supervision that included feedback from the monitoring process was provided. Visit checklists designed to promote protocol adherence were completed by the clinicians during each visit.

All Medical Management visits were audiorecorded, and $5 \%$ of each clinician's visits were rated for treatment fidelity, defined as competence on 6 dimensions. Comprehensive descriptions of the dimensions and the rating procedures can be found in Petinnati et al. ${ }^{11}$ Briefly, the dimensions are (1) completion 
of essential elements of the Medical Management intervention (protocol); (2) no deviations from the protocol (strict adherence); (3) conveying professionalism, expertise, and confidence in treatment (authoritative),

(4) communicating correct educational information in a clear and concise manner (informative); (5) being warm, friendly, compassionate, helpful, and concerned (warmth); and (6) providing structure, coherence, and flow to the visit (direction). Each dimension was rated on a scale ranging from 1 to 7 , with a rating of 5 or higher defined as "competent." Overall, based on the ratings of 671 Medical Management visits, the intervention was delivered with high fidelity, ie, ratings of 5 or more on all 6 dimensions for most visits.

\section{Study Measures}

Patient variables included the number of Medical Management visits, total number of minutes with the treating clinicians, the number of different clinicians seen by a patient during the course of treatment, and patient reports of therapeutic alliance and satisfaction with treatment. Patients completed the Working Alliance Inventory, a standard measure of the therapeutic relationship modified to address the Medical Management intervention, after the 4 th visit. (This instrument, as well as the public dataset, will be available at the NIAAA Web site by January 2009.) Patients also completed a Satisfaction with Treatment questionnaire at the end of treatment. Responses to 3 questions about Medical Management were summed, with lower scores indicative of a greater degree of satisfaction.

Clinician-level variables include the level of professional training (bachelor of nursing, advanced medical training, physician) and the number of patients for which the clinician had primary responsibility. Patients were assigned to the clinician who conducted their initial Medical Management visit. The adherence ratings from the COMBINE Training and Monitoring Center were used to calculate mean adherence levels, which are indicative of the overall style and pattern of delivery, for each clinician.

Measures of drinking, derived from a calendarbased assessment that provides an estimate of daily alcohol consumption, were assessed at baseline and monthly during treatment. ${ }^{14,15}$ Within-treatment means for the percentage of days the patient was completely abstinent from alcohol and the percentage of days in which the patient engaged in heavy drinking were used in this analysis. Drinking measures were combined with a standard measure of alcohol-related problems to create a dichotomous measure of clinical improvement defined as abstinent or moderate drinking without problems. Improvement was assessed at the end of treatment, and patients with missing data were considered to have no clinical improvement. More detailed definitions of outcome measures were reported in the main outcome report. ${ }^{7,16}$

\section{Statistical Analysis}

Statistical analyses were done using SAS version 8 (SAS Institute Inc, Cary, NC). Hierarchical linear modeling is an extension of the general linear model that allows the variance in outcome variables to be analyzed at multiple levels. The adherence data collected in the COMBINE study supported analysis at the patient and clinician levels. Variance in outcome was partitioned into variance attributable to patients and variance attributable to clinicians. Statistical models were compared using the Akaike's Information Criterion suggested by Singer ${ }^{17}$ as the most useful of the fit statistics. It is formulated by SAS so that smaller numbers mean a better fit.

A series of exploratory bivariate and simple regression analyses were conducted to determine the value of adding clinician level variables to the model containing the patient level variables. Measures of clinician performance were included in the hierarchical model only if there was a consistent and significant pattern in the regressions and between outcome measures.

\section{RESULTS}

Of the 1,226 patients randomized to receive the Medical Management intervention, 1,162 were assigned to 37 clinicians for whom adherence ratings were available. This subsample was used in the hierarchical analysis. As shown in Table 1, the patients participated in $87 \%$ of the Medical Management intervention, with slightly more than 3 hours of total time. There was an increase of 15 days of abstinence and a drop in 15 days of heavy drinking per month during treatment. At the end of treatment 667 (57\%) showed clinical improvement. Most patients saw only 1 clinician (72\%, 835). The number of clinicians seen by a patient was correlated with clinical improvement $(r=.08047 ; P=.006)$ but not with the drinking outcomes. Overall, patients who remained in treatment reported a good alliance or therapeutic relationship with their clinicians and were satisfied with their treatment.

The 37 clinicians with adherence ratings were assigned to a mean of 32 patients (SD 25; range, 1100). Fifty-one percent, or 19 , of the clinicians had a bachelor's degree in nursing, an additional 6 nurses had advanced practice degrees, 10 were physicians, 1 was a pharmacist, and 1 was a physician's assistant. The level of professional training and the number of patients assigned to a clinician were not significantly correlated with any of the patient outcome measures (data not shown). Based on the exploratory analysis, the moni- 


\begin{tabular}{|c|c|c|c|c|c|}
\hline Variable & $\mathbf{N}$ & Mean & SD & Min & Max \\
\hline Medical management visits & 1,162 & 7.8 & 2.4 & 1 & 17 \\
\hline Medical management clinicians seen & 1,162 & 1.3 & 0.5 & 1 & 3 \\
\hline $\begin{array}{l}\text { Patient's total time spent in Medical } \\
\text { Management visits, min }\end{array}$ & 1,162 & 185 & 64 & 30 & 450 \\
\hline $\begin{array}{l}\text { Patient Working Alliance Inventory score } \\
4 \text { weeks into Medical Management } \\
\text { treatment }{ }^{\mathrm{a}}\end{array}$ & 972 & 65.86 & 8.56 & 24 & 84 \\
\hline $\begin{array}{l}\text { Patient end-of-treatment satisfaction with } \\
\text { Medical Management score }{ }^{b}\end{array}$ & 923 & 5.7 & 1.4 & 3 & 15 \\
\hline $\begin{array}{l}\text { Days abstinent in each of months } 1-4 \text { of } \\
\text { treatment, mean \% }\end{array}$ & 1,086 & 76 & 28 & 0 & 100 \\
\hline $\begin{array}{l}\text { Days heavy drinking in each of months } \\
1-4 \text { of treatment, mean } \%\end{array}$ & 1,086 & 15 & 22 & 0 & 100 \\
\hline
\end{tabular}

The results of the clinician-adherence variables were consistent across the 3 outcome variables. The patient predictors accounted for the greatest proportion of variance in outcome $(96 \%$ for days abstinent, $98 \%$ for days of heavy drinking, and 99\% for clinical improvement). For percentage of days abstinent, there was a small but significant proportion of variance attributable to clinician factors after entering the patient variables. For all 3 outcome variables, adding the clinician factors, strict adherence, and authoritative dimensions accounted for a significant proportion of the unexplained clinician variance (days of abstinence, $\rho=.36$; days

toring dimensions used in the hierarchical analyses were authoritative $($ mean $=6.0$, SD .14 ; range $=5.6-6.4)$, and there was strict adherence to the study protocol $\left(\right.$ mean $=5.9 ; \mathrm{SD} .11_{i}$ range $\left.=5.6-6.0\right)$. These ratings indicate that all the clinicians were deemed competent in their delivery of Medical Management, as defined by these dimensions.

Hierarchal linear modeling required complete data on each patient and clinician. The limiting factor in the analysis was the completion of the working alliance and treatment satisfaction measures. The resulting sample sizes ( $\mathrm{n}=813$ for drinking outcomes, $\mathrm{n}=797$ for improvement) represent significant selection bias. The patients who did not have complete data were significantly younger and more likely to be of minority status. They did not differ on their pretreatment drinking measures, but they had poorer drinking outcomes, and fewer improved clinically.

The hierarchal linear modeling results, displayed in Table 2, were consistent across the 3 outcome variables listed in the table, with a few exceptions. The most consistent variables were the number of visits attended (associated with good outcomes) and the total number of minutes spent in Medical Management visits (associated with poor outcomes). Pretreatment measures of all 3 outcomes were predictive of end-of-treatment outcomes.

Higher ratings on the Working Alliance Inventory scale were associated with more abstinence and less heavy drinking during treatment, but they did not predict end-of-treatment clinical improvement. Satisfaction with treatment at the end of the intervention was significantly associated with increased abstinence and better clinical improvement, but not with a decrease in heavy drinking. of heavy drinking, $\rho=.52$; clinical improvement $\rho=.35$ ) and resulted in the lowest Akaike's Information Criterion value, indicating the best fitting model. There were significant main effects for both measures in the abstinence and heavy drinking models. A higher mean score on the authoritative dimension in combination with a lower mean score on the strict adherence dimension was associated with more positive outcomes. Adding either dimension to the model by itself (without also adding the other dimension) did not have a significant effect on the model. For clinical improvement only the authoritative score was significant when the strict adherence measure remained in the model.

\section{DISCUSSION}

This study examined the relationship of patient and clinician factors in the delivery of the Medical Management intervention with treatment outcomes in patients participating in the COMBINE study. Patients attending more visits reduced their drinking and had a better clinical outcome. Patients who were doing less well had fewer visits, but because the visits tended to be lengthy, these patients had more face-to-face clinician time than those patients with better outcomes. The patient's perception of a good clinician-patient relationship during treatment predicted better drinking outcomes. Patients satisfied with Medical Management treatment upon completion reported more abstinence and were more likely to have clinical improvement.

The context of the Medical Management intervention is important in interpreting the results of the clinician dimensions that were related to positive outcomes. This consistently monitored intervention 


\begin{tabular}{|c|c|c|c|}
\hline Outcome & $\begin{array}{l}\text { Parameter } \\
\text { Estimate }\end{array}$ & SE & $P$ Value \\
\hline \multicolumn{4}{|l|}{ Days abstinent, mean \% } \\
\hline Intercept & 76.8294 & 1.4001 & $<.0001$ \\
\hline Clinician mean authoritative score & 34.6004 & 13.7917 & .0172 \\
\hline Clinician mean strict adherence score ${ }^{a}$ & -40.1780 & 15.1000 & .0119 \\
\hline Patient number of visits & 4.3219 & .7739 & $<.0001$ \\
\hline Patient total minutes in Medical Management visits & -.0555 & .01974 & .0014 \\
\hline Patient baseline days abstinent, $\%$ & .2164 & .0350 & $<.0001$ \\
\hline $\begin{array}{l}\text { Patient working alliance score for Medical } \\
\text { Management }\end{array}$ & .3541 & .1102 & .0014 \\
\hline $\begin{array}{l}\text { Patient satisfaction score with Medical Manage- } \\
\text { ment treatment }\end{array}$ & -2.1368 & .7120 & .0028 \\
\hline \multicolumn{4}{|l|}{ Days heavy drinking, \% } \\
\hline Intercept & 14.5775 & .8941 & $<.0001$ \\
\hline Clinician mean authoritative score & -18.4035 & 8.9239 & .0471 \\
\hline Clinician mean strict adherence score ${ }^{a}$ & 28.5826 & 9.6938 & .0058 \\
\hline Patient number of visits & -4.0094 & .5618 & $<.0001$ \\
\hline Patient total minutes in Medical Management visits & .04592 & .01403 & .0011 \\
\hline Patient baseline days heavy drinking, $\%$ & .09392 & .02252 & $<.0001$ \\
\hline $\begin{array}{l}\text { Patient working alliance score for Medical } \\
\text { Management }\end{array}$ & -.2223 & .08151 & .0065 \\
\hline 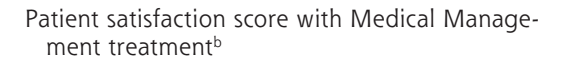 & .9113 & .5258 & .0835 \\
\hline \multicolumn{4}{|l|}{ Clinical improvement } \\
\hline Intercept & .6256 & .02301 & $<.0001$ \\
\hline Clinician mean authoritative score & .5687 & .2297 & .0186 \\
\hline Clinician mean strict adherence score & -.3890 & .2480 & .1263 \\
\hline Patient number of visits & .06682 & .01475 & $<.0001$ \\
\hline Patient total minutes in Medical Management visits & -.00126 & .0004 & .0006 \\
\hline Patient baseline clinical composite index & -.08436 & .03681 & .0222 \\
\hline $\begin{array}{l}\text { Patient working alliance score for Medical } \\
\text { Management }\end{array}$ & .002176 & .002138 & .3091 \\
\hline $\begin{array}{l}\text { Patient satisfaction score with Medical Manage- } \\
\text { ment treatment }\end{array}$ & -.02877 & .01391 & .0389 \\
\hline
\end{tabular}

to convey optimism for recovery in the face of discouraging news from the patient. This observation is consistent with the literature on the effect of clinicians' expectancies on patient outcomes. ${ }^{18}$ There is also evidence reported on the importance of a clinician conveying a diagnosis and treatment plan in an authoritative manner. ${ }^{19}$

The strict adherence dimension was designed to measure the extent to which the clinician adheres to the protocol during the visit. Competency ratings overall indicated that the clinicians did not significantly drift from the protocol. Informal discussion with the monitors about dialogues with high ratings (closer to 7 than 5) on the strict adherence dimension indicated that these ratings reflected situations in which the clinician stayed with the protocol no matter what other issues the patient raised. The clinicians who achieved the highest mean scores, while competent in delivering the protocol, might have achieved better outcomes with their patients had they also shown some flexibility to address unique patient concerns when they arose. These findings mean that some flexibility in delivering Medical Management, based on good clinical judgment and in conjunction was delivered by medically trained clinicians who underwent a modest training program to deliver the intervention. Overall, there was very little variation in delivery and clinician competence, indicating that the clinicians were warm, informative, provided direction, and followed and completed the protocol. Furthermore, results suggested that clinicians contributed to improving patient outcomes if they more easily conveyed confidence in the treatment (high ratings on the authoritative dimension), and showed some flexibility in the delivery (minimum competence ratings-scores closer to 5 than 7 -on the strict adherence dimension).

Although patient-clinician dialogues were not evaluated for content, monitors who rated dialogues as high on authoritativeness anecdotally conveyed that these ratings reflected intervention clinicians who were able with optimism and hope for recovery, supports better outcomes with the intervention.

One limitation of this study is that this intervention was provided in a research environment rather than a clinical practice setting. Though designed for portability to primary care, it will be crucial to examine that assumption. A future research question is whether outcomes would be similar if the Medical Management intervention were delivered in a setting with less control over the delivery of the intervention and with fewer resources to follow up with patients. These results imply that in primary care practice it may be feasible to delegate medical management for patients on naltrexone to nurses or pharmacists as members of the treatment team-how to integrate this team approach into primary care is fertile ground for implementation research. 
Additionally, these patients were seeking treatment, and as such, they may differ from the usual primary care patient with alcohol dependence, who may or may not want to talk about their alcohol problem. Finally, the 6 dimensions of clinician performance that were used in this analysis are not exhaustive and may not be the most important. These dimensions were not derived from an extensive review of the literature. Rather, they were formulated from clinical principles held by the COMBINE study's monitoring center, whose expertise is in evaluating process variables in psychosocial treatments. In addition, these 6 dimensions were thought to reflect general measures of clinician competence and fidelity in delivering the Medical Management intervention.

The Medical Management intervention plus prescribing naltrexone was shown to be successful in the recovery of alcohol dependence in the large, multisite COMBINE study. This treatment regimen is thought to be consonant with the skills of medically trained clinicians and requires minimal additional training (no specialty behavioral training) to treat patients with alcohol problems. There is still a high level of social stigma associated with requesting and receiving treatment for alcohol problems. Using effective medications and managing the treatment in a primary care setting with a medically based intervention, such as Medical Management, can substantially decrease the stigma and increase the availability of treatment to patients who would normally go without such care.

To read or post commentaries in response to this article, see it online at http://www.annfammed.org/cgi/content/full/6/5/435.

Key words: Alcoholism/drug therapy; medication therapy management; naltrexone; primary care

Submitted May 31, 2007; submitted, revised, March 22, 2008; accepted March 28, 2008.

Funding support: Preparation of this report was supported in part by grants U10 AA011756 (RDW), U10 AA11787 and K05 AA014715 (SSO), U10 AA011721 (JDH), U10 AA11715 (JSL), and U10 AA11777 (RS) from the National Institute on Alcohol Abuse and Alcoholism, and grant K24 DA022288 (RDW) from the National Institute on Drug Abuse.

Acknowledgments: The reported data were collected as part of the multisite COMBINE Study sponsored by the National Institute on Alcohol Abuse and Alcoholism (NIAAA). We thank Harold Delaney, PhD, of the University of New Mexico for his support and contribution to the statistical analyses conducted for this report. Further information about study site and other publications from the COMBINE Study can be found at http://www.cscc.unc.edu/COMBINE. A full listing of COMBINE Study staff is available at http://www.cscc.unc.edu/COMBINE.

\section{References}

1. Samet JH, Friedmann P, Saitz R. Benefits of linking primary medical care and substance abuse services. Arch Intern Med. 2001;161(1):85-91.
2. Friedmann PD, Zhang Z, Hendrickson J, Stein MD, Gerstein DR. Effect of primary medical care on addiction and medical severity in substance abuse treatment programs. J Gen Intern Med. 2003;18(1):1-8.

3. Willenbring $\mathrm{ML}$, Olson $\mathrm{DH}$. A randomized trial of integrated outpatient treatment for medically ill alcoholic men. Arch Intern Med. 1999;159(16):1946-1952.

4. Weisner C, Mertens J, Parthasarathy S, Moore C, Lu Y. Integrating primary medical care with addiction treatment: a randomized controlled trial. JAMA. 2001;286(14):1715-1723.

5. Saxon AJ, Malte CA, Sloan KL, et al. Randomized trial of onsite versus referral primary medical care for veterans in addictions treatment. Med Care. 2006;44(4):334-342.

6. O'Malley SS, Rounsaville BJ, Farren C, et al. Initial and maintenance naltrexone treatment for alcohol dependence using primary care vs. specialty care: a nested sequence of 3 randomized trials. Arch Intern Med. 2003;163(14):1695-1704.

7. Anton RF, O'Malley SS, Ciraulo DA, et al. Combined pharmacotherapies and behavioral interventions for alcohol dependence. The COMBINE Study: a randomized controlled trial. JAMA. 2006;295(17):2003-2017.

8. Combine Study Research Group. Testing combined pharmacotherapies and behavioral interventions in alcohol dependence: rationale and methods. Alcohol Clin Exp Res. 2003;27(7):1107-1122.

9. COMBINE Study Research Group. Testing combined pharmacotherapies and behavioral interventions for alcohol dependence (The COMBINE Study): a pilot feasibility study. Alcohol Clin Exp Res. 2003;27(7):1123-1131.

10. Pettinati HM, Weiss RD, Miller WR, Donovan D, Ernst DB, Rounsaville BJ. Medical Management Treatment Manual: A Clinical Research Guide for Medically Trained Clinicians Providing Pharmacotherapy as Part of the Treatment for Alcohol Dependence. Bethesda, MD: National Institute on Alcohol Abuse and Alcoholism; 2004.

11. Pettinati HM, Weiss RD, Dundon W, et al. A structured approach to medical management (MM): a psychosocial intervention to support pharmacotherapy in the treatment of alcohol dependence. J Stud Alcohol Suppl. 2005;(15):170-178.

12. Connors GJ, Carroll KM, DiClemente CC, Longabaugh R, Donovan DM. The therapeutic alliance and its relationship to alcoholism treatment participation and outcome. J Consult Clin Psychol. 1997;65(4):588-598.

13. Donovan DM, Kadden RM, DiClemente CC, Carroll KM. Client satisfaction with three therapies in the treatment of alcohol dependence: results from Project MATCH. Am J Addict. 2002;11(4):291-302.

14. Miller WR. (1996). Form 90: A Structured Assessment Interview for Drinking and Related Behaviors (Test Manual). Bethesda, MD: National Institute on Alcohol Abuse and Alcoholism.

15. Miller WR, Del Boca FK. Measurement of drinking behavior using the Form 90 family of instruments. J Stud Alcohol Suppl. 1994;12 (Dec):112-118.

16. Zweben A, Cisler RA. Clinical and methodological utility of a composite outcome measure for alcohol treatment research. Alcohol Clin Exp Res. 2003;27(10):1680-1685.

17. Singer JD. Using SAS PROC MIXED to fit multilevel models, hierarchical models, and individual growth models. J Educ Behav Stat. 1998;23(4):323-355.

18. Crow R, Gage H, Hampson S, Hart J, Kimber A, Thomas H. The role of expectancies in the placebo effect and their use in the delivery of health care: a systematic review. Health Technol Assess. 1999;18:1-96.

19. Scovern AW. From placebo to alliance: the role of common factors in medicine. In: Hubble MA, Duncan BL, Miller SD, eds. The Heart and Soul of Change. Washington DC: American Psychological Association; 1999:259-296. 